\title{
Diagnosis of MV Oil Filled Cable Terminations with X-Ray Imaging and Infrared (IR) Thermography
}

\author{
${ }^{1}$ Mohd Rafi Adzman, ${ }^{1}$ Ismail Daut, ${ }^{1}$ Muzamir Isa, ${ }^{1}$ Baharuddin Ismail, ${ }^{1}$ Omar Aliman \\ ${ }^{2}$ Matti Lehtonen and ${ }^{2}$ Petri Hyvonen ${ }^{2}$ \\ ${ }^{1}$ School of Electrical System Engineering, KUKUM Malaysia \\ ${ }^{2}$ Laboratory of Power System, TKK Finland
}

\begin{abstract}
A main purpose of doing a diagnostic in cable system including its accessories such as impregnated paper insulated oil filled cable termination is to predict failure before they occur. Those cable and accessories, which are about to fail, can be replaced, thereby reducing the incidence of electrical system failure, thus reducing the electricity outages. This paper presents the work carried out to find any possible method that can be used to detect a drying out of oil impregnated paper tape cable terminations. Furthermore, the diagnosis has to be used without destroying the terminations. Two diagnosis methods; infrared (IR) thermography and X-ray imaging are reviewed and the diagnosis result is presented. By comparison of two-diagnosis method in this work, the X-ray imaging method is the best approach to observe and detect a drying out of paper tape oil impregnated cable terminations.
\end{abstract}

Key words: IR Thermography, X-ray imaging, insulation and cable diagnosis

\section{INTRODUCTION}

The background of this work is a diagnosis of a medium voltage (MV) oil filled cable terminations. In general, terminations are required when MV cables are connected to the overhead lines or other electrical apparatus such as transformer, bushing, capacitor bank, circuit breaker and so on. Cable termination is one of the important components in the electrical distribution power system. It is designed to possess the same integrity as their associated cables.

Normally, before the evolution of heat shrink and cold shrink cable terminations, porcelain type of termination as shown in Fig. 1 and rubber tube type of termination were the two major types of cable termination used for impregnated paper insulated cables. This type of cable terminations is normally oil filled. One of the defects of this type of cable termination is the lack of insulation oil inside it. In the worst case if it becomes totally empty and at the same time there is no approach that can help to detect it. This defect is probably due to the poor sealing, leakage and a local damage which is caused from improper installation ${ }^{[1,2]}$. Other possible reason for drying up is insulation oil penetration into the paper insulation and further into the conductor. The dried up of insulation oil inside the termination will lead to the abnormal heat distribution as well as abnormal voltage distribution in the termination itself and further will lead to a cable failure. Normally it will take time before the insulation oil is totally dried up.

The main purpose of doing a diagnostic in cable system including its accessories such as impregnated

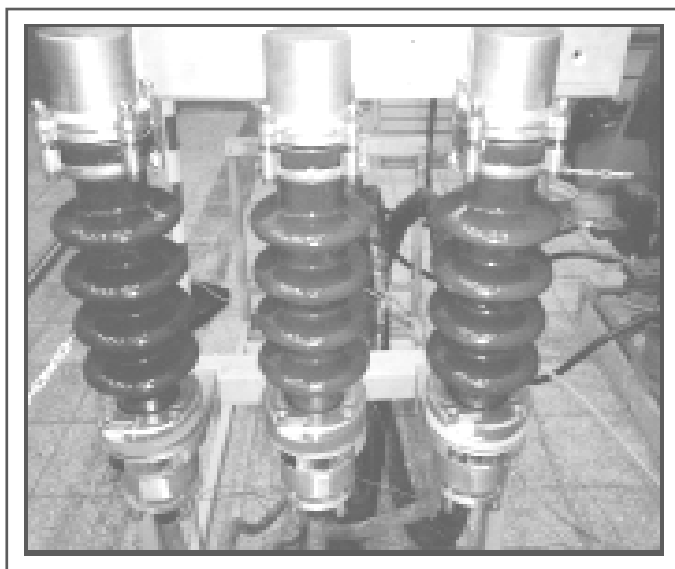

Fig. 1: Example of oil filled porcelain type of termination

paper insulated oil filled cable termination is to predict failure before they occur. Those cable and accessories which are about to fail can be replaced provided that if we can detect them earlier thereby reducing the incidence of electrical system failure, thus reducing the electricity outages.

\section{MATERIALS AND METHODS}

Two techniques are discussed these are infrared (IR) thermography and X- ray imaging.

Infrared thermography: Infrared (IR) imaging technique is one of the diagnostic tools that we proposed to detect the dried up of oil filled cable termination in this project. 
The purpose of this experiment was to observe the temperature distribution of cable termination by using the IR imaging camera. We want to observe the temperature characteristic at the termination which has different level of oil insulation.

During experiment, three unit of porcelain type of termination as shown in Fig. 1 has been used. To identify whether there is a difference in temperature distribution due to low level of oil insulation inside the cable termination, one of the insulation oil from the three units of the termination has been removed to half level and the other two was remained in full level. From Fig. 1, the termination in the middle was the one that we removed its insulation oil to half level. The setup in the laboratory is shown as in Fig. 2. It mainly consisted of 3-phase paper impregnated lead covered (PILC) cable with the porcelain type of termination at the end of the cable. Current to the PILC cable and it termination is supplied by a $415 \mathrm{~V}, 50 \mathrm{~Hz} 3$-phase AC supply through a current transformer. In order to control the voltage and the current, 3-phase variac which was connected to the 3-phase main AC supply has been used. The current was measured by using 3 unit of current transformer (CTs) and measured signals were fed to the digital precision wattmeter.

The maximum AC current that was fed to the cable and its termination was 200A per phase. During the experiment, the cable and its termination were heated around 2 hours. A versatile IR imaging camera model 760 from Inframetrics was used for thermal imaging measurements. The IR camera observed the temperature at the termination continuously from beginning till the end of the experiment for the period of two hour.

$\mathrm{X}$-ray imaging: X-ray imaging is one of the latest and most advanced methods for non destructive test (NDT) methodologies. Mostly X-ray has been used for decades in medical application. Nowadays, its scope of services has been established for all kind of applications such as in aerospace, automotive, electrical, electronic, petrochemical, welding and also general inspection. The concept of digital X-ray imaging system configuration is shown in Fig. 3.

One of the advantages of this kind of advanced technology is portable with a special feature of low Xray radiation and hence less dangerous to human and easy to handle ${ }^{[3]}$. By using this technique we can see the internal fault and from that reason it is easy to make a decision about the necessity of replacing or maintaining the termination

\section{RESULTS AND DISCUSSION}

Infrared thermography: After carrying out the measurements for two hours the IR digital camera images were analyzed. The IR digital camera imaging result are shown in Fig. 4.

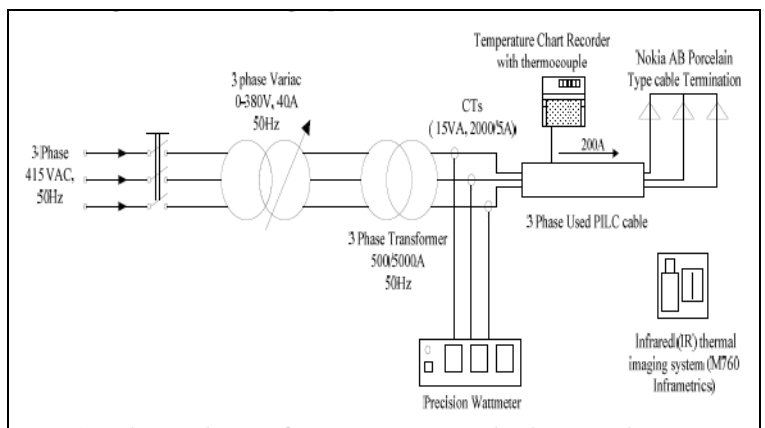

Fig. 2: Schematic diagram of measuring temperature distribution in cable termination with the infrared (IR) digital camera

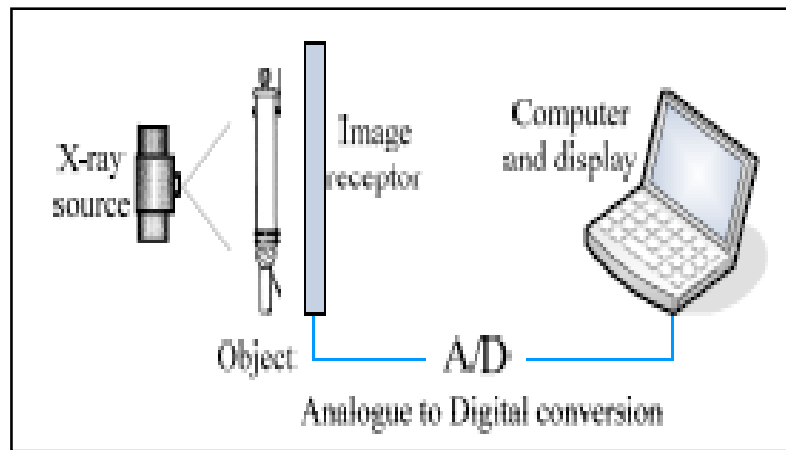

Fig. 3: Example of digital X-ray imaging system configuration

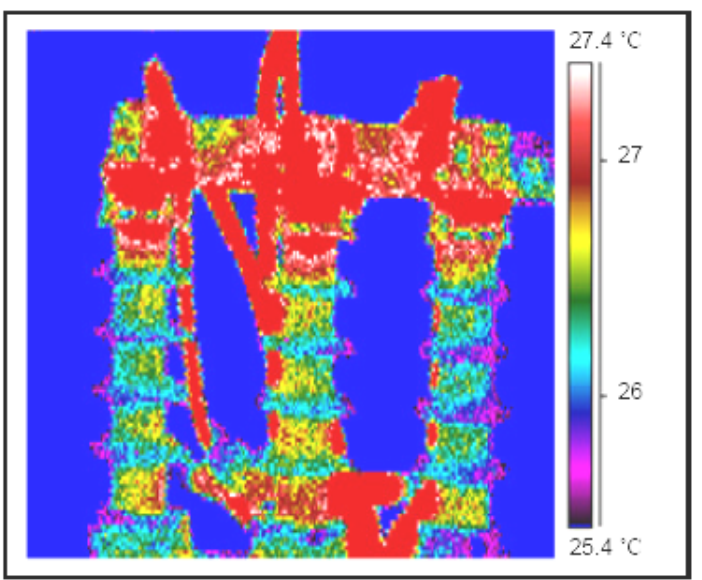

Fig. 4: Thermal images of porcelain type of cable termination subjected at $200 \mathrm{~A}, 50 \mathrm{~Hz}$ for 120 $\min$.

It can be seen that the middle porcelain termination having most of the area with yellowiest color compared with the other two terminations. Red color can be seen at the lower and upper part of the middle porcelain termination. Based on IR thermal image results, we can know that the middle porcelain termination having higher temperature than the other two terminations.

The unsatisfactory part from this experiment, we could not see any separator mark of oil level showing a temperature difference between two levels for instant upper and lower part of termination. 


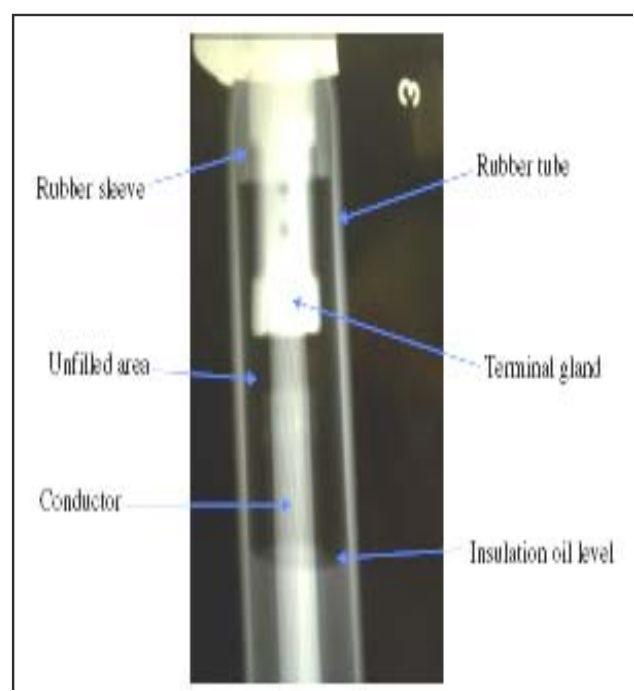

(a)

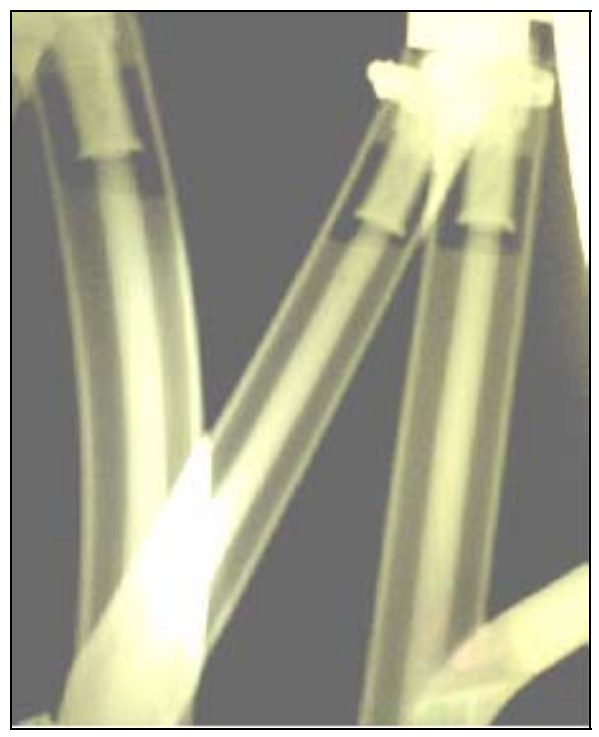

(b)

Fig. 5a and b: X-ray image on the upper side of rubber tube type of termination

Most probably, this kind of fault can only be seen by using a high sensitivity and resolution infrared thermography camera. The experiments also focus on porcelain type of cable termination. Further investigation using IR camera measurements should be made to other type of oil filled cable termination such as rubber tube type of cable termination. The diagnosis results are expected to be different due to different material properties of the termination.

$\mathrm{X}$-ray imaging: The X-ray image of rubber tube cable termination was taken at several existing substations in Finland. From the X-ray image as shown in Fig. 5b, it can clearly be seen that the oil is always in horizontal position, even though the cable termination posture was not in vertical position. The X-ray image shows that the insulation oil inside the rubber tube type of termination is at the specified mark level. Based on the X-ray image we can make a fast judgment that as long as the insulation oil inside the termination remains in full position, the termination is in good condition. Otherwise if there is some other internal defect that can cause partial discharges in the cable termination system. In case of porcelain type of termination, it required higher quantity of X-ray to produce a good image of shadows. This is because porcelain has higher density and it absorbed more X-rays than rubber.

\section{CONCLUSION}

In general, the investigation of external faults is much simpler than the internal faults. Admittedly, to analyze an internal fault like a drying out of paper tape cable termination is not an easy task and it is difficult to be analysed by common electrical test. Based on the investigation, practice and experiment results with IR thermography diagnosis to the cable termination with low insulation oil level gave unsatisfactory result. This is probably due to the limitations of the infrared system such low resolution, unsuitable waveband and required good interpretation. In most cases the infrared technique is best for hunting the hot spot in cable system such as loose contact or connection of conductor. The X-ray imaging diagnostic method seems to be the best approach to observe and detect a drying out of oil impregnated paper tape oil filled cable terminations. The X-ray images show clearly the level of insulation oil inside the rubber tube termination. From the X-ray image, we can make a fast decision about the necessary replacing or maintaining the termination before it can cause further damage and create a fault to the cable network.

\section{REFERENCES}

1. Niancang, H., 1998. The infrared thermography diagnostic technique of high voltage electrical equipment with internal faults. IEEE Proc. Power System Technol., 1: 110-115.

2. Taylor, B., G. Morrison and D. Welch, 1997. Evaluating medium voltage cable splices and termination. IEEE Conf. Paper on Petroleum and Chemical Industry Society, pp: 121-127.

3. De Mello, D.R., E. Acioli and N. Gois, 2004. Location of internal faults in high voltage lines and distribution equipment. Transmission and Distribution Conference and Exposition: Latin America, IEEE/PES, pp: 281-28. 